hep-ph/9902312

$\mathrm{IC} / 99 / 9$

\title{
Testing the Solar Neutrino Conversion with Atmospheric Neutrinos
}

\author{
O. L. G. Peres ${ }^{1}$ and A. Yu. Smirnov ${ }^{1,2}$ \\ (1) The Abdus Salam International Centre for Theoretical Physics, I-34100 Trieste, Italy \\ (2) Institute for Nuclear Research of Russian Academy of Sciences, Moscow 117312, \\ Russia
}

\begin{abstract}
Neutrino oscillations with parameters $\Delta m_{\odot}^{2}=(2-20) \cdot 10^{-5} \mathrm{eV}^{2}, \sin ^{2} 2 \theta_{\odot}>0.65$, relevant for large mixing MSW solution of the solar neutrino problem can lead to an observable (up to 10 - $12 \%$ ) excess of the e-like events in the sub-GeV atmospheric neutrino sample. The excess has a weak zenith angle dependence in the low energy part of the sample and strong zenith angle dependence in the high energy part. The excess rapidly decreases with energy of neutrinos, it is suppressed in the multi-GeV sample. These signatures allow one to disentangle the effect of the oscillations due to solar $\Delta m^{2}$ from other possible explanations of the excess. The up-down asymmetry of the excess may change the sign with energy being positive in the sub-GeV region and negative in the multi-GeV range. Predicted properties of the excess are in agreement with the SuperKamiokande data.
\end{abstract}




\section{Introduction}

The SuperKamiokande (SK) collaboration [1], 2] continues to accumulate the data which strengthen the evidence of the muon neutrino oscillations. Whole variety of the data can be fitted well assuming $\nu_{\mu} \leftrightarrow \nu_{\tau}$ channel with the maximal or close to maximal mixing:

$$
\Delta m_{a t m}^{2}=(1.5-8) \times 10^{-3} \mathrm{eV}^{2}, \quad \sin ^{2} 2 \theta_{a t m}>0.8
$$

At the same time, there are some facts which indicate that oscillations of the atmospheric neutrino is not reduced completely to two neutrino case and the electron neutrino is involved in the oscillations too. The $\nu_{\mu} \leftrightarrow \nu_{e}$ oscillations as the sub-leading mode are possible and probably desired.

- The data shows an excess of the e-like events in the sub-GeV sample. The SK collaboration accounts the excess by up-scaling the overall normalization of the neutrino

fluxes. However, recent cosmic ray measurements [3] indicate that possible increase of the neutrino fluxes was probably overestimated. Moreover, it seems that the excess depends on the neutrino energy (which is impossible to explain by overall normalization). The largest excess is in the low energy part of the sample $(p<0.4 \mathrm{GeV})$ and it is smaller in the high energy part.

- There are some indications of the up-down asymmetry of the e-like events which changes with energy. The asymmetry is positive in the low momenta region and it tends to be negative at high energies (multi-GeV range).

- The data show strong zenith angle dependence of the $\mu$-like events which imply relatively small oscillation effect for down-going neutrinos. In the two neutrino framework it is difficult to reconcile this fact with rather low value of the double ratio $R_{\mu / e} \equiv(\mu / e) /(\mu / e)_{M C}$ integrated over zenith angle [ [A]. (Although recent data give higher $R_{\mu / e}$ the problem is not completely settled down.) In the case of three neutrino oscillations the $\nu_{\mu}$-signal can be further suppressed and the $\nu_{e}$-signal enhanced. 
There have been a number of studies of the atmospheric neutrino oscillations with the three (or more) neutrino mixing [5, 6, 7, 8, 9, 10, 11. Most of them where performed in the framework of the so called "one level dominating scheme" when the mass splitting between two lightest states, $\Delta m_{21}^{2}$, is neglected. In this case the oscillations in the subleading channel occur due to mixing of the electron neutrino in the heaviest mass state with mass splitting $\Delta m_{a t m}^{2}$. The effects of the sub-leading channel are restricted by the CHOOZ result [12]. They are reduced to vacuum oscillations for the sub-GeV sample. For the multi-GeV sample the Earth matter effect becomes important which may enhance or suppress the oscillations [10].

In several papers all mass splittings have been taken into account. In Ref. [1] generic $3 \nu$ effects have been considered with relatively large $\Delta m_{21}^{2}$ for lightest states (outside the region of solar neutrino solutions). In Ref. [13] the attempts have been made to explain the zenith angle dependence of the $\mu$-like events by the $\nu_{\mu} \leftrightarrow \nu_{e}$ oscillations with small $\Delta m_{21}^{2} \sim 10^{-4} \mathrm{eV}^{2}$ and large mixing. The channel $\nu_{\mu} \leftrightarrow \nu_{\tau}$ with large $\Delta m_{32}^{2} \sim 0.2 \mathrm{eV}^{2}$ implied by the LSND result leads to overall suppression of the signal. It was marked that for $\Delta m_{21}^{2}<10^{-4} \mathrm{eV}^{2}$ the matter of the Earth significantly suppresses the oscillation effect. In this scheme one expects also strong zenith angle dependence of the e-like events which contradicts the SK data.

It was marked in Ref. [14] that the effect of the sub-leading oscillations driven by $\Delta m_{\odot}^{2}$ responsible for the solar neutrino deficit is significant only for the sub-GeV events and the size of effect at the level of the statistical errors. In Ref. [15] it was argued that the excess of the e-like events in the sub-GeV sample favors large mixing MSW solution of the solar neutrino problem. However analysis of 535 days of the SuperKamiokande data in Ref. [16] leads to conclusion that there is no difference between the large and small mixing solutions.

The impact of the solar neutrino sector on the atmospheric neutrino fluxes has been also studied in the context of exact parity model [17]. 
In this paper we will study in details the effects of oscillations of the atmospheric neutrinos with parameters $\left(\Delta m_{\odot}^{2}, \sin ^{2} 2 \theta_{\odot}\right)$ of the large mixing MSW solution of the solar neutrino problem. The analysis of recent data (including the spectral and zenith angle information) leads to the following values [2, 18]

$$
\Delta m_{\odot}^{2}=(2-20) \cdot 10^{-5} \mathrm{eV}^{2}, \quad \sin ^{2} 2 \theta_{\odot}=0.65-0.95
$$

This region corresponds to two neutrino mixing. It is slightly modified if there is an admix-

ture of $\nu_{e}$ flavor in the third state which satisfies the CHOOZ bound [12]: $\left|U_{e 3}\right|^{2}=\sin ^{2} \theta_{13}<$ 0.05 .

There are two motivations of present study:

(1). Recent SK data give some indications in favor of the large mixing MSW solution (flat distortion of the recoil electron spectrum, day-night asymmetry, flat zenith angle distribution of the night signal, etc.) [2, 19].

(2). Studies of the atmospheric neutrinos enter now new stage of precision measurements when the data become sensitive to sub-leading effects.

The paper is organized as follows. In Sec. 2 we find general expressions for the atmospheric neutrino fluxes in presence of the three neutrino oscillations. In Sec. 3 we discuss properties of relevant two neutrino probabilities. In sect. 4 we calculate the excess of the e-like events for different energy ranges and study its properties. In sect. 5 we consider influence of the sub-leading oscillations on the $\mu$-like events and $R_{\mu / e}$. In Sec. 6 we discuss the results and perspectives.

\section{Three-flavor oscillations of atmospheric neutrinos}

We consider the three-flavor neutrino system with hierarchical mass squared differences: $\Delta m_{21}^{2}=\Delta m_{\odot}^{2}<<\Delta m_{31}^{2}=\Delta m_{a t m}^{2}$ (see Eqs. (1, 2, 2) ). The evolution of the neutrino vector of 
state $\nu_{f} \equiv\left(\nu_{e}, \nu_{\mu}, \nu_{\tau}\right)^{T}$ is described by the equation

$$
i \frac{d \nu_{f}}{d t}=\left(\frac{U M^{2} U^{\dagger}}{2 E}+V\right) \nu_{f},
$$

where $E$ is the neutrino energy and $M^{2}=\operatorname{diag}\left(0, \Delta m_{21}^{2}, \Delta m_{31}^{2}\right)$ is the diagonal matrix of neutrino mass squared eigenvalues. $V=\operatorname{diag}\left(V_{e}, 0,0\right)$ is the matrix of matter-induced neutrino potentials with $V_{e}=\sqrt{2} G_{F} N_{e}, G_{F}$ and $N_{e}$ being the Fermi constant and the electron number density, respectively. The mixing matrix $U$ is defined through $\nu_{f}=U \nu_{m}$, where $\nu_{m}=\left(\nu_{1}, \nu_{2}, \nu_{3}\right)^{T}$ is the vector of neutrino mass eigenstates. It can be parameterized as $U=U_{23} U_{13} U_{12}$. The matrix $U_{i j}=U_{i j}\left(\theta_{i j}\right)$ performs the rotation in the $i j$ - plane by the angle $\theta_{i j}$. We have neglected possible CP-violation effects in the lepton sector which are suppressed in the case of the mass hierarchy.

Let us introduce new states $\tilde{\nu}=\left(\nu_{e}, \tilde{\nu}_{2}, \tilde{\nu}_{3}\right)^{T}$ obtained by performing the $U_{23}$ - rotation: $\nu_{f}=U_{23} \tilde{\nu}$. The Hamiltonian $\tilde{H}$ that describes the evolution of the vector $\tilde{\nu}$ can be found from Eq. (3):

$$
\tilde{H}=\frac{1}{2 E} U_{13} U_{12} M^{2} U_{12}^{\dagger} U_{13}^{\dagger}+V
$$

Let us assume that mixing of the electron neutrino in the heavy state is negligible, '] so that $U_{13} \approx 1$. In this case we get explicitly

$$
\tilde{H} \approx\left(\begin{array}{ccc}
s_{12}^{2} \Delta m_{21}^{2} / 2 E+V_{e} & s_{12} c_{12} \Delta m_{21}^{2} / 2 E & 0 \\
s_{12} c_{12} \Delta m_{21}^{2} / 2 E & c_{12}^{2} \Delta m_{21}^{2} / 2 E & 0 \\
0 & 0 & \Delta m_{31}^{2} / 2 E
\end{array}\right)
$$

$\left(c_{12} \equiv \cos \theta_{12}, s_{12} \equiv \sin \theta_{12}\right.$, etc.) According to Eq. (幽, the $\tilde{\nu}_{3}$ state decouples from the rest of the system and evolves independently. Therefore the S-matrix (the matrix of amplitudes) in the basis $\left(\nu_{e}, \tilde{\nu}_{2}, \tilde{\nu}_{3}\right)$ has the following form :

$$
S \approx\left(\begin{array}{ccc}
A_{e e} & A_{e 2} & 0 \\
A_{2 e} & A_{22} & 0 \\
0 & 0 & A_{33}
\end{array}\right)
$$

\footnotetext{
${ }^{1}$ We comment of the effect of this mixing in Section 6. Results of the detailed studies will be published elsewhere [20].
} 
where

$$
A_{33}=\exp \left(-i \phi_{3}\right), \quad \phi_{3}=\frac{\Delta m_{31}^{2} L}{2 E},
$$

and $L$ is the total distance traveled by the neutrinos. The $\left(\nu_{e}, \tilde{\nu}_{2}\right)$ subsystem evolves according to the $2 \times 2$ Hamiltonian $\left(\nu_{e}-\tilde{\nu}_{2}\right.$ sub-matrix in Eq. (44)). The latter depends on the potential $V_{e}$, mixing angle $\theta_{12}$ and the mass squared difference $\Delta m_{21}^{2}$. Let us denote by

$$
P_{2} \equiv\left|A_{e 2}\right|^{2}=\left|A_{2 e}\right|^{2}=1-\left|A_{e e}\right|^{2}=1-\left|A_{22}\right|^{2}
$$

the probability of the $\nu_{e} \leftrightarrow \tilde{\nu}_{2}$ oscillations. For antineutrinos we have $\bar{P}_{2}=P_{2}\left(-V_{e}\right)$.

The $S$-matrix in the flavor basis can be obtained from Eq. (河) by $U_{23}$-rotation: $U_{23} S U_{23}^{\dagger}$. It gives the probabilities of flavor oscillations as $P\left(\nu_{\alpha} \rightarrow \nu_{\beta}\right)=\left|\left(U_{23} S U_{23}^{\dagger}\right)_{\alpha \beta}\right|^{2}$. The appropriate probabilities equal

$$
\begin{gathered}
P\left(\nu_{e} \rightarrow \nu_{e}\right)=1-P_{2}, \\
P\left(\nu_{e} \leftrightarrow \nu_{\mu}\right)=P\left(\nu_{\mu} \leftrightarrow \nu_{e}\right)=c_{23}^{2} P_{2}, \\
P\left(\nu_{\mu} \rightarrow \nu_{\mu}\right)=1-c_{23}^{4} P_{2}-2 s_{23}^{2} c_{23}^{2}\left[1-\sqrt{1-P_{2}} \cos \phi\right],
\end{gathered}
$$

where

$$
\phi \equiv \phi_{3}-\phi_{2}
$$

is the phase difference of the amplitudes $A_{22}$ and $A_{33}: \phi_{2} \equiv \arg A_{22}$ is the phase of $A_{22} ; \phi_{3}$ is defined in Eq. (6).

Using the probabilities given in Eqs. (8 10$)$ one can find modifications of the atmospheric neutrino fluxes due to the oscillations. Let $F_{e}^{0}$ and $F_{\mu}^{0}$ be the electron and muon neutrino fluxes at the detector in the absence of oscillations. Then the fluxes in the presence of oscillations can be written as

$$
\begin{gathered}
F_{e}=F_{e}^{0}\left[1+P_{2}\left(r c_{23}^{2}-1\right)\right], \\
F_{\mu}=F_{\mu}^{0}\left[1-\frac{c_{23}^{2}}{r}\left(r c_{23}^{2}-1\right) P_{2}-\frac{1}{2} \sin ^{2} 2 \theta_{23}\left(1-\sqrt{1-P_{2}} \cos \phi\right)\right],
\end{gathered}
$$


where

$$
r\left(E, \Theta_{\nu}\right)=\frac{F_{\mu}^{0}\left(E, \Theta_{\nu}\right)}{F_{e}^{0}\left(E, \Theta_{\nu}\right)}
$$

is the ratio of the original muon and electron neutrino fluxes. Here $\Theta_{\nu}$ is the neutrino zenith angle.

For antineutrinos $P_{2}$ should be substituted by $\bar{P}_{2}$ in Eqs (8 - 12). The solution of the solar neutrino problem implies that the resonance is in the neutrino channel, therefore the mixing and the transition probability are smaller for antineutrinos: $P_{2}>\bar{P}_{2}$.

As follows from Eq. (11) the effect of oscillations on the electron neutrino flux is proportional to the factor $\left(r c_{23}^{2}-1\right)$. Therefore one can have either an excess or a deficiency of the e-like events depending on values of $r$ and $c_{23}$. The ratio $r$ depends both on the zenith angle and on the neutrino energy. For $r=2$ which corresponds to the sub-GeV sample, there will be an excess of e-like events for $\theta_{23}<45^{\circ}$ and a deficiency for $\theta_{23}>45^{\circ}$. The SK best fit is $\theta_{23}=45^{\circ}$; in this case there would be no deviation from the prediction for $r=2$. In the multi-GeV range $r$ is typically $3-3.5$ in the vertical direction with averaged over zenith angle value about 2.5 .

\section{The transition probability}

We have calculated the two neutrino transition probabilities $P_{2}, \bar{P}_{2}$ using the parameterization of the distribution of density in the Earth from Ref. [21]. For the analysis of the results, it is worthwhile to consider transitions of neutrinos in the Earth as oscillations in medium which consists of several layers with constant densities. This, in fact, gives rather good approximation to the exact results.

The depth and the length of oscillations are determined by

$$
\sin ^{2} 2 \theta_{m}=\sin ^{2} 2 \theta_{12}\left(\frac{\Delta m_{21}^{2}}{2 E \Delta H}\right)^{2}, \quad l_{m}=\frac{2 \pi}{\Delta H},
$$


where

$$
\Delta H=\sqrt{\left(\cos 2 \theta_{12} \frac{\Delta m_{21}^{2}}{2 E}-V_{e}\right)^{2}+\left(\sin 2 \theta_{12} \frac{\Delta m_{21}^{2}}{2 E}\right)^{2}}
$$

is the level splitting (difference between the eigenvalues of $H$ ). The resonance energy equals

$$
E_{R}=\frac{\Delta m_{21}^{2} \cos 2 \theta_{12}}{2 V_{e}}=0.17 \mathrm{GeV}\left(\frac{\Delta m_{21}^{2}}{5 \cdot 10^{-5} \mathrm{eV}^{2}}\right)\left(\frac{2.0 \mathrm{~g} / \mathrm{cm}^{3}}{Y_{e} \rho}\right) \cos 2 \theta_{12} .
$$

In the mantle, for typical value $\Delta m_{21}^{2}=5 \cdot 10^{-5} \mathrm{eV}^{2}$ and for $\sin ^{2} 2 \theta_{12}=0.8$ we get $E_{R}=0.08 \mathrm{GeV}$ which is below the threshold of the sub-GeV range. Therefore for

$\Delta m_{21}^{2}<5 \cdot 10^{-5} \mathrm{eV}^{2}$ the oscillations are in the matter dominated regime when the potential is substantially larger than the kinetic term: $V \gg \Delta m^{2} / 2 E$. In this case, the depth of oscillations is roughly proportional to $\left(\Delta m^{2}\right)^{2}$, and the oscillation length, $l_{m}$, is close to the refraction length, $l_{0}$, and only weakly depends on the energy:

$$
\sin ^{2} 2 \theta_{m} \sim \sin ^{2} 2 \theta_{12}\left(\frac{\Delta m_{21}^{2}}{2 E V_{e}}\right)^{2}, \quad l_{m} \approx l_{0}=\frac{2 \pi}{V_{e}} .
$$

For the multi-GeV range the approximation of Eq. (16) works for $\Delta m^{2}$ as big as $10^{-4} \mathrm{eV}^{2}$.

We find that the maximal neutrino oscillation effect in the mantle is achieved at $\cos \Theta_{\nu} \sim$ -0.35 and the effect is zero at $\cos \Theta_{\nu} \sim-0.6$. For $\cos \Theta_{\nu}<-0.84$ neutrinos cross both the mantle and the core of the Earth. The interplay of the oscillations in the mantle and in the core leads to some enhancement of the transition probability in spite of larger density of the core. The oscillation effects in the antineutrino channel are smaller by factor $2-3$.

The expressions of the Eq. (16) are valid for small $\sin ^{2} 2 \theta_{m}$. With increase of $\Delta m^{2}$, the increase of $\sin ^{2} 2 \theta_{m}$, and consequently, the probabilities is slowing down. In the neutrino channel the depth approaches one in the resonance. In the antineutrino channel the mixing also increases but it is always below vacuum mixing.

\section{Excess of the e-like events}

In what follows we will calculate the dependences of the excess of e-like events on the zenith angle of electron, $\Theta_{e}$. The general expression for the number of e-like events, $N_{e}$ as a function 
of $\Theta_{e}$ is

$$
\begin{aligned}
& N_{e} \propto \sum_{\nu \bar{\nu}} \int d E_{\nu} d E_{e} d\left(\cos \Theta_{\nu}\right) d h \quad F_{e}\left(E_{\nu}, \Theta_{\nu}\right) \frac{d \sigma}{d E_{e}} \\
& \Psi\left(\Theta_{e}, \Theta_{\nu}, E_{\nu}\right) \kappa_{e}\left(h, \cos \Theta_{\nu}, E_{\nu}\right) \varepsilon\left(E_{e}\right),
\end{aligned}
$$

where $F_{e}$ is the atmospheric $\nu_{e}$-flux at the detector given in Eq. (11) (the fluxes $F_{e}^{0}$ and $F_{\nu}^{0}$ without oscillations are taken from Ref. [22]); $d \sigma / d E_{e}$ are the differential cross sections taken from Ref. [23], $\kappa_{e}$ is the normalized distribution of neutrino production points, $h$ is the height of production, $\epsilon\left(E_{e}\right)$ is the detection efficiency of the electron, $\Psi$ is the "dispersion" function which describes deviation of lepton zenith angle from the neutrino zenith angle ( For details see Ref. [24]).

Notice that the integration over the neutrino zenith angle and neutrino energy leads to a significant smearing of the $\Theta_{\nu}$ dependence. The average angle between the neutrino and the outgoing charged lepton is almost $60^{\circ}$ in the sub-GeV range and it is about $15^{\circ}-20^{\circ}$ in the multi-GeV region. Neutrinos and antineutrinos of a given flavor are not distinguished in the atmospheric neutrino experiments, so that the neutrino and antineutrino signals are summed in Eq. (18) which also leads to weakening of the oscillation effect.

According to Eq. (12) and Eq. (18) the relative excess of the e-like events, $\epsilon_{e}$, can be represented as

$$
\epsilon_{e} \equiv \frac{N_{e}}{N_{e}^{0}}-1 \approx \bar{P}\left(\Theta_{e}\right)\left(\bar{r}\left(\Theta_{e}\right) c_{23}^{2}-1\right),
$$

where $\bar{P}\left(\Theta_{e}\right)$ is the probability averaged over appropriate energy and zenith angle intervals as well as over neutrinos and antineutrinos; $\bar{r}\left(\Theta_{e}\right)$ is the effective ratio of the electron and muon neutrino fluxes for a given energy and angle intervals.

The up-down asymmetry is given by

$$
A_{e}^{U / D}=\frac{N_{e}^{U}-N_{e}^{D}}{N_{e}^{U}+N_{e}^{D}},
$$




\begin{tabular}{|c|c|c|}
\hline$\Delta m_{21}^{2}\left(\mathrm{eV}^{2}\right)$ & $\bar{\epsilon}_{e}(0.8)(\%)$ & $\bar{\epsilon}_{e}(0.8)(\%)$ \\
\hline & $\sin ^{2} 2 \theta_{12}=0.65$ & $\sin ^{2} 2 \theta_{12}=0.90$ \\
\hline $1.10^{-5}$ & 0.1 & 0.2 \\
\hline $4.10^{-5}$ & 1.8 & 2.0 \\
\hline $8.10^{-5}$ & 4.7 & 4.8 \\
\hline $2.10^{-4}$ & 8.6 & 9.3 \\
\hline
\end{tabular}

Table 1: The excess integrated over $\Theta_{e}, \bar{\epsilon}_{e}$, for different choices of $\Delta m_{21}^{2}$ and $\sin ^{2} 2 \theta_{12}$.

where

$$
N_{e}^{U}=\int_{-1.0}^{-0.2} d \cos \Theta_{e} N_{e}\left(\Theta_{e}\right), \quad N_{e}^{D}=\int_{0.2}^{1.0} d \cos \Theta_{e} N_{e}\left(\Theta_{e}\right),
$$

and $N_{e}\left(\Theta_{e}\right)$ are given in Eq. (18). This definition includes both asymmetry of the original neutrino flux (in particular, due to geomagnetic effect) and the asymmetry due to oscillations. The asymmetry due to oscillations only can be estimated as

$$
A_{\text {osc }}^{U / D} \approx \frac{\epsilon_{e}^{u p}-\epsilon_{e}^{\text {down }}}{2},
$$

where $\epsilon_{e}^{U}$ and $\epsilon_{e}^{D}$ are values of the excess integrated over same zenith angle bins as in Eq. (21).

Let us consider the sub-GeV events which correspond to the limit of integration in Eq. (18), $p<1.33 \mathrm{GeV}$. In Fig. 1 we show the zenith angle dependences of the excess of the e-like events for $\sin ^{2} 2 \theta_{12}=0.9, \sin ^{2} \theta_{23}=0.8$ and different values of $\Delta m_{21}^{2}$. The following remarks are in order.

(i). The excess increases rapidly with $\Delta m_{21}^{2}$ in correspondence with behaviour of the probability. In the first vertical upward bin the excess can reach $12.3 \%$ for $\Delta m_{21}^{2}=2 \cdot 10^{-5}$ $\mathrm{eV}^{2}$. The excess integrated over all bins for different values of $\Delta m_{21}^{2}$ is shown in the Table 1 .

(ii). The excess has rather weak zenith angle dependence. For instance, the updown asymmetry equals $A_{\text {osc }}^{U / D}=1.5-4.0 \%$ for $\Delta m_{21}^{2} \gtrsim 10^{-4} \mathrm{eV}^{2}, \sin ^{2} 2 \theta_{12}=0.9$ and 
$\sin ^{2} \theta_{23}=0.8$

(ii). The excess depends very weakly on the solar mixing angle $\sin ^{2} 2 \theta_{12}$ (see Fig. 2 and Table 1). This is related to weak dependence of $\sin ^{2} 2 \theta_{m}$ on $\sin ^{2} 2 \theta_{12}$ in the region of parameters under consideration. The decrease of $\sin ^{2} 2 \theta_{12}$ is compensated by the shift of the resonance to larger energies.

(iv). The excess increases with the decrease of $\sin ^{2} 2 \theta_{23}$. According to Eq. (19) the dependence of the excess on the mixing angle of leading channel, $\theta_{23}$ is determined by the factor $\left(\bar{r}\left(\Theta_{e}\right) c_{23}^{2}-1\right)$. Therefore for arbitrary value of $\theta_{23}$ the excess can be found using $\epsilon_{e}(0.8)$ (the calculated excess for $\sin ^{2} 2 \theta_{23}=0.8$; see Table 1 ):

$$
\epsilon_{e}\left(\sin ^{2} 2 \theta_{23}\right)=\epsilon_{e}(0.8) \cdot \frac{c_{23}^{2} \bar{r}\left(\Theta_{e}\right)-1}{0.723 \bar{r}\left(\Theta_{e}\right)-1} \approx \epsilon_{e}(0.8) \cdot \frac{\cos 2 \theta_{23}}{0.45} .
$$

For $\sin ^{2} 2 \theta_{23}=0.7,0.9,0.95$ and $\bar{r}=2$ the excess (in the units of $\epsilon_{e}(0.8)$ ) equals 1.23, 0.716, 0.49 correspondingly. The excess disappears when the mixing in the leading channel approaches the maximal one. Notice, however that even for $\sin ^{2} 2 \theta_{23}=0.95$ an appreciable excess still survives.

The excess decreases with increase of energy of the selected events. This corresponds to decrease of the mixing parameter in medium: $\sin ^{2} 2 \theta_{m} \propto 1 / E_{\nu}^{2}$ in the matter dominated range. In Fig. 3 and Fig. 4 we show the excess of the e-like events (as the function of the zenith angle $\Theta_{e}$ ) for the low energy part of the sub-GeV sample with the electron momentum $p<0.4 \mathrm{GeV}$, and for the high energy part of the sample with $p>0.4 \mathrm{GeV}$.

For the low energy sample (Fig. 3) the excess can reach $12 \%$ in the upward vertical bin for $\Delta m_{21}^{2}=2 \cdot 10^{-4} \mathrm{eV}^{2}$. The excess has weak zenith angle dependence, e.g. for $\Delta m_{21}^{2}=10^{-4} \mathrm{eV}^{2}$ it decreases from $9 \%$ to $7.6 \%$ with increases of the zenith angle .

The integrated excess $\bar{\epsilon}_{e}$ equals $11.3 \%$ and $8.4 \%$ for the two indicated values of $\Delta m_{21}^{2}$.

In the high energy sample (Fig. 4) the excess in the vertical bin also reaches $12 \%$ $\left(\Delta m_{21}^{2}=210^{-4} \mathrm{eV}^{2}\right)$, but it decreases rapidly with $\Delta m_{21}^{2}$. In this sample the zenith angle dependence is very strong, e.g. for $\Delta m_{21}^{2}=10^{-4} \mathrm{eV}^{2}$ the excess decreases from $5 \%$ in the 
vertical upward bin to $0.7 \%$ in the vertical down going bin, the asymmetry $A_{o s c}^{U / D}=2-5 \%$ for $\Delta m_{21}^{2} \gtrsim 10^{-4} \mathrm{eV}^{2}$. The integrated excess is smaller than in the low energy sample: $\bar{\epsilon}_{e}=$ $6 \%$ and $2.5 \%$ for $\Delta m_{21}^{2}=2 \cdot 10^{-4} \mathrm{eV}^{2}$ and $10^{-4} \mathrm{eV}^{2}$ respectively.

Thus, with increase of energy the up-down asymmetry becomes more profound. However the absolute value of the excess decreases.

Let us now consider the multi-GeV events. The zenith angle distributions of the excess for different values of $\Delta m_{21}^{2}$ are shown in Fig. 4. Let us compare the effects in the multi-GeV and sub-GeV samples:

(i) Typical energy of neutrinos which produce multi - GeV events, $E_{m G} \sim 3-4 \mathrm{GeV}$, is 4 - 5 times larger than the energy, $E_{s G}$, in the sub-GeV sample. Therefore the mixing parameter and the probability are suppressed by factor $\left(E_{m G} / E_{s G}\right)^{2} \sim 16-25$.

(ii) The ratio of the fluxes, $r$, for the multi-GeV range is about $r \approx 3$ (for upward bin), so that the value of factor $\bar{r}\left(\Theta_{e}\right) c_{23}^{2}-1$ turns out to be $2-3$ times larger than in the sub-GeV sample.

(iii) Since the average angle between the neutrino and produced charge lepton is smaller in the multi-GeV range, the averaging effect is smaller. In particular, in the vertical bin the relative contribution of trajectories which cross the core of the Earth is larger. The transition probability for the core crossing trajectories is slightly enhanced.

(iv) As the result of the interplay of these factors, the excess in the multi-GeV range is 5 - 7 times smaller than in the sub-GeV region.

The zenith angle dependence of the excess is stronger. The excess decreases with $|\cos \Theta|$ and it disappears for the horizontal bin. The up-down asymmetry due to oscillations can reach $A_{\text {osc }}^{U / D}=2 \%$ for $\Delta m_{21}^{2}=2 \cdot 10^{-4} \mathrm{eV}^{2}$ and it decreases rapidly with $\Delta m_{21}^{2}$. For $\Delta m_{21}^{2}=10^{-4} \mathrm{eV}^{2}$ the excess and the asymmetry are below $1 \%$. 


\section{$5 \mu$-like events and the Double Ratio}

In contrast to electron neutrinos, the muon neutrinos have both small and large $\Delta m^{2}$ modes of oscillations. According to Eq. (12) the flux can be written as

$$
\frac{F_{\mu}}{F_{\mu}^{0}}=1-\sin ^{2} 2 \theta_{23} \sin ^{2} \frac{\phi}{2}-\epsilon_{\mu}-\epsilon_{i n t},
$$

where the first two terms correspond to the standard $\nu_{\mu} \leftrightarrow \nu_{\tau}$ survival probability with slightly modified phase (see below);

$$
\epsilon_{\mu}=\frac{c_{23}^{2}}{r}\left(r c_{23}^{2}-1\right) P_{2} \approx \frac{c_{23}^{2}}{r} \epsilon_{e}
$$

is the correction due to $\nu_{\mu}-\nu_{e}$ oscillations and

$$
\epsilon_{\text {int }}=\left(1-\sqrt{1-P_{2}}\right) \sin ^{2} 2 \theta_{23} \cos \phi \approx \frac{1}{4} P_{2} \sin ^{2} 2 \theta_{23} \cos \phi
$$

is the term which describes the interference of the oscillations with large and small $\Delta m^{2}$. The phase $\phi$ can be estimated in the limit of matter dominance as

$$
\phi \approx\left(\Delta m_{31}^{2}+s_{12}^{2} \Delta m_{21}^{2}\right) \frac{L}{2 E}
$$

The first term here is the standard vacuum oscillation phase, whereas the second term is the correction due to $3 \nu$ mixing. For the sub-GeV sample this correction can be neglected. Indeed, in the upward bins the oscillations due to $\Delta m_{31}^{2}$ are averaged and an additional small contribution from the second term (associated to $\Delta m_{21}^{2}$ ) play no role. For downward bins with the average distance $L \sim 100 \mathrm{~km}$ the second term is negligible.

Due to strong averaging effect the correction $\epsilon_{\text {int }}$ can be neglected. As the result, the number of $\mu$-like events can be written as

$$
N_{\mu}=N_{\mu}^{0}\left[\bar{P}\left(\nu_{\mu} \leftrightarrow \nu_{\tau}\right)-\bar{\epsilon}_{\mu}\right]
$$

where $\bar{P}$ is the averaged (over energy and zenith angle) two neutrino probability. For maximal mixing the correction $\bar{\epsilon}_{\mu}$ is very small. With decrease of $\sin ^{2} 2 \theta_{23}$ the survival 
probability increases $\Delta P \sim 1 / 2 \Delta \sin ^{2} 2 \theta_{23}$. At the same time, the correction $\bar{\epsilon}_{\mu}$ increases too, thus partly compensating of $\Delta P$. The compensation effect depends strongly on $\Delta m_{21}^{2}$ and $\sin ^{2} 2 \theta_{23}$. For $\Delta m_{21}^{2}=10^{-4} \mathrm{eV}^{2}$ and $\sin ^{2} 2 \theta_{23}=0.8$ we find $\bar{\epsilon}_{\mu}=2 \%$, whereas $\Delta P=10 \%$. For $\sin ^{2} 2 \theta_{23}=0.9$ the corresponding numbers are $\bar{\epsilon}_{\mu}=1.5 \%$ and $\Delta P=5 \%$.

The double ratio $R_{\mu / e} \equiv\left(N_{\mu} / N_{e}\right)\left(N_{\mu}^{0} / N_{e}^{0}\right)$ can be written as

$$
R_{\mu / e}=R_{\mu / e}^{\max } \frac{1-0.5 \sin ^{2} 2 \theta_{23}-\bar{\epsilon}_{\mu}}{1+\bar{\epsilon}_{e}}
$$

where $R_{e / \mu}^{\max }$ is the double ratio of two neutrino oscillations with maximal mixing. In the

double ratio both corrections $\bar{\epsilon}_{\mu}$ and $\overline{\epsilon_{e}}$ compensate the decrease $\sin ^{2} 2 \theta_{23}$. For $\sin ^{2} 2 \theta_{23}=0.9$ we find $\Delta P=5 \%, \bar{\epsilon}_{\mu}=1.5 \%$ and $\bar{\epsilon}_{e}=3 \%$, so that the total increase of the double ration is very small: $0.5 \%$.

The corrections $\bar{\epsilon}_{\mu}$ and $\bar{\epsilon}_{e}$ are substantially smaller in the multi-GeV range, where one would expect $R_{\mu / e}^{m G}>R_{\mu / e}^{s G}$.

\section{Discussion and Conclusions}

We have considered the oscillation effects in the atmospheric neutrinos induced by $\Delta m_{21}^{2}$ and $\sin ^{2} 2 \theta_{21}$ from the region of large mixing MSW solution of the solar neutrino problem.

The oscillations can lead to the observable excess of the e-like events in the sub-GeV sample with the following properties. The maximal excess is in the low energy part of the sub-GeV sample: the integrated excess can range from $12 \%$ to $3 \%$ for $\Delta m^{2}$ decreasing from $2 \cdot 10^{-4}$ to $4 \cdot 10^{-5} \mathrm{eV}^{2}$. The effect decreases with increase of energy of the sample. For high energy part of the sub-GeV range the integrated excess can reach $6 \%$. For the multi-GeV sample the excess is below $1.5 \%$.

Notice that the excess of the e-like events can also be due to oscillations induced by the large $\Delta m_{31}^{2}$ responsible for the leading channel of the atmospheric neutrino oscillations, provided that there is some admixture of the $\nu_{e}$ - flavor in the $\nu_{3}$ state. The two effects differ 
by the energy dependence. The excess due to large $\Delta m^{2}$ oscillations increases with energy: the excess should be substantially stronger in the multi-GeV sample [10]. The latest SK data show stronger effect in the sub-GeV sample (especially in the upward going bins) thus preferring the solar $\Delta m^{2}$ effect.

The excess due to the solar $\Delta m^{2}$ oscillations has certain zenith angle dependences: The up-down asymmetry due to oscillations is very weak in the low energy part of the sub-GeV sample, and it is strong in the high energy part of the sample. In the multi-GeV range the asymmetry is also strong, however the excess itself is much weaker.

These properties will allow one to distinguish the oscillation effect from the normalization of fluxes (especially in future high statistics and high precision experiments).

The excess depends on the mixing angle $\sin ^{2} 2 \theta_{23}$ responsible for the leading channel of oscillations. The effect decreases with $\sin ^{2} 2 \theta_{23}$ and it is strongly suppressed for maximal mixing, $\sin ^{2} 2 \theta_{23}=1$. Therefore, it is impossible to exclude the large mixing MSW solution from the atmospheric neutrino data or even put some bounds on parameters, unless the $\sin ^{2} 2 \theta_{23}$ will be measured with a good precision. On the contrary, if the excess with described properties will be established, this will both confirm the large mixing MSW solution and show that $\sin ^{2} 2 \theta_{23}$ differs from 1 .

In this paper we discussed the excess of the e-like events. For $\theta_{23}>45^{\circ}$ one expects the suppression of the e-like events which, in fact, disfavored by the present data. Therefore, if $\Delta m_{21}^{2}$ will be further restricted by the solar neutrino observations, one will be able to put the bound on $\sin ^{2} 2 \theta_{23}$.

Comparison of the predicted excess with data (Fig. 1 - 5) shows reasonable agreement. For $\Delta m_{21}^{2} \sim 10^{-4} \mathrm{eV}^{2}$ the excess due to oscillations reproduces both the size and the zenith angle distribution of the observed excess in the sub-Gev range. It also gives reasonable description of the data in the low energy part of the sub-GeV sample. At the same time, the predicted excess is smaller than the detected one in the high energy sub-GeV range, and also in the multi-GeV range. Notice, however, that in the high energy sub-GeV range, 
and especially in multi-GeV range, the effects of the large $\Delta m_{31}^{2}$ (neglected here) can be important [10]. They can change the signal by $15 \%$ in the multi-GeV sample and by 5 - $6 \%$ in the high energy part of the sub-GeV sample. Also non-trivial interference of small and large $\Delta m^{2}$ oscillation effects is possible [20]. It is clear however, that with present statistics it is impossible to make definite conclusions. In fact, it could be that the explanation of the data will require some interplay of the normalization of fluxes and different oscillation effects.

The excess has the positive up-down asymmetry. The positive asymmetry at low energies can be reconciled with the negative asymmetry at high energies (multi-GeV sample) indicated by the present data. Indeed, the excess due to solar $\Delta m^{2}$ (with positive asymmetry) decreases with increase of energy and in the multi-GeV range the dominant effect will be due to oscillations with large $\Delta m^{2}$ and nonzero $\theta_{13}$. In this case the sign of the excess is determined by factor [10]:

$$
\left(r s_{23}^{2}-1\right)
$$

(with $s_{23}^{2}$ instead of $c_{23}^{2}$, see Eq. (11)). In the multi-GeV range the average ratio of the fluxes $r \approx 2.5$, and for $s_{23}^{2}=0.277$ we get negative value of the factor (30): -0.3 . Indeed, the latest SK data indicate a negative up-down asymmetry at high energies. The interpretation of the multi-GeV zenith angle distribution can require some interplay of the normalization which explains the excess of events in the down-going bins $(\cos \Theta=0.2 \div 1)$ and the oscillations which suppress the number of events in the upward going bins $(\cos \Theta=-1 \div-0.2)$.

Further studies of solar neutrinos (in particular, searches for recoil electron spectrum distortion and the day-night effect) will allow one to prove or disprove the large mixing MSW solution. Observations of significant day-night effect will imply small values of $\Delta m_{21}^{2}$ and therefore small excess of the e-like events. On the contrary, weak day-night effect and upturn of the distortion of the recoil electron spectrum at low energies will testify for large $\Delta m_{21}^{2}$ and large excess. The KAMLAND experiment [25] will test whole the range of the large mixing angle solution. 
If the effects discussed in this paper will be confirmed we can be left with the "bi-large mixing scheme" with large (but not maximal) mixing between neighboring generations and small $e-\tau$ mixing. The mass hierarchy will be rather weak: $m_{2} / m_{3} \sim 0.1-0.3$ which will allow one to explain large mixing without special arrangements.

\section{Note added}

After this work had been accomplished the paper [26] has appeared in which the implications of the low-energy SK data for LMA solution of the solar neutrino problem are discussed. It is claimed that oscillations lead always to decrease of number of e-like events in comparison with no oscillation case. This statement contradicts our results. The error

follows from using the ratio of numbers of events, $R_{\mu / e}^{M C}$, instead of ratio of the neutrino fluxes ( $r$ in our notation) in formulas (2.22)-(3.9) of Ref. [26]. Also matter effects must be included.

\section{Acknowledgments}

This work was supported by DGICYT under grant PB95-1077 and by the TMR network grant ERBFMRXCT960090 of the European Union. A. Yu. S. thanks Fundacion Bilbao Viscaya (FBBV) for supporting his visit to Valencia when our collaboration started. O.L.G. Peres thanks to M.C. Gonzalez-Garcia for the atmospheric neutrino code and for the extensive joint work made in collaboration with H. Nunokawa.

\section{References}

[1] SuperKamiokande Collaboration, Y. Fukuda et al., Phys. Lett. B433, 9 (1998);idem, B436, 33 (1998); Phys. Rev. Lett. 81, 1562 (1998). 
[2] SuperKamiokande Collaboration, Y. Suzuki et al, talk given at 17th International Workshop on Weak Interactions and Neutrinos (WIN '99), 24-30 January 1999 , Cape Town, South Africa.

[3] T. Sanuki, Talk at the Symposium "New Era in Neutrino Physics" (Satellite Symposium after "Neutrino'98"), Tokyo, June 1998.

[4] J. M. LoSecco, hep-ph/9807359.

[5] J. Pantaleone, Phys. Rev. D49, 2152 (1994); G. L. Fogli, E. Lisi, D. Montanino, Astropart. Phys. 4, 177 (1995); G. L. Fogli, E. Lisi, D. Montanino, G. Scioscia, Phys. Rev. D55, 4385 (1997); G. L. Fogli, E. Lisi, A. Marrone, Phys. Rev. D57, 5893 (1998); O. Yasuda, Phys. Rev. D58, 091301(1998).

[6] C. Giunti, C. W. Kim, J. D. Kim, Phys. Lett. B352, 357 (1995); P. F. Harrison, D. H. Perkins, Phys. Lett. B349, 137 (1995); Phys. Lett. B396, 186 (1997); H. Fritzsch, Z.-Z. Xing, Phys. Lett. B372, 265 (1996); C. Giunti, C. W. Kim, M. Monteno, Nucl. Phys. B 521, 3 (1998); R. Foot, R. R. Volkas, O. Yasuda, Phys. Lett. B421, 245 (1998) ; Phys. Lett. B433, 82(1998).

[7] G. L. Fogli, E. Lisi, A. Marrone, D. Montanino, Phys. Lett. B425, 341 (1998).

[8] G. L. Fogli, E. Lisi, D. Montanino, Phys. Rev. D49, 3626 (1994); G. L. Fogli, E. Lisi, G. Scioscia, Phys. Rev. D52, 5334 (1995); S. M. Bilenkii, C. Giunti, C. W. Kim, Astropart. Phys. 4, 241 (1996); O. Yasuda, H. Minakata, hep-ph/9602386; Nucl.Phys.B 523, 597 (1998); Phys. Rev. D56, 1692 (1997) (hep-ph/9712291); T. Teshima, T. Sakai, O. Inagaki, hep-ph/9801276; T. Teshima, T. Sakai, hep-ph/9805386; V. Barger, S. Pakvasa, T. J. Weiler, K. Whisnant, Phys. Lett. B437, 107(1998); R. Barbieri, L. J. Hall, D. Smith, A. Strumia, N. Weiner, hep-ph/9807235; V. Barger, T. J. Weiler, K. Whisnant, Phys. Lett. B440 1(1998). 
[9] J.J. Gomez-Cadenas and M.C. Gonzalez-Garcia, Z. Phys. C71, 443(1996); V. Barger, T.J. Weiler and K. Whisnant, Phys. Lett. B427, 97(1998); V. Barger, S. Pakvasa, T.J. Weiler, K. Whisnant Phys. Rev. D58, 093016(1998).

[10] E. Kh. Akhmedov, A. Dighe, P. Lipari, A. Yu. Smirnov, hep-ph/9808270 to appear in Nucl. Phys. B .

[11] O. Yasuda, hep-ph/9602342; hep-ph/9707564.

[12] CHOOZ Collaboration, M. Apollonio et al., Phys.Lett. B420, 397 (1998).

[13] R.P. Thun and S.Mckee, Phys. Lett. B439, 123(1998).

[14] G. L. Fogli, E. Lisi, A. Marrone and G. Scioscia, Phys. Rev. D59, 033001 (1998).

[15] C. W. Kim, U. W. Lee, Phys. Lett. B444, 204(1998).

[16] T. Teshima, T. Sakai, hep/ph 9901219.

[17] J. Brunn, R. Foot and R. R. Volkas, Phys. Lett. B413 (1997) 109.

[18] J.N. Bahcall, P.I. Krastev and A.Yu. Smirnov, Phys. Rev. D58, 096016 (1998).

[19] J.N. Bahcall, P.I. Krastev and A.Yu. Smirnov, in preparation.

[20] O.L.G.Peres and A.Yu. Smirnov, work in progress.

[21] E. Lisi and D. Montanino, Phys. Rev. D56, 1792 (1997).

[22] V. Agrawal et al., ibid D53, 1314 (1996); T. K. Gaisser and T. Stanev, ibid D57, 1977 (1998).

[23] P. Lipari, M. Lusignoli, and F. Sartogo, Phys. Rev. Lett. 74, 4384 (1995).

[24] M. C. Gonzalez-Garcia, H. Nunokawa, O. L. G. Peres, T. Stanev, J. W. F. Valle, Phys. Rev. D58, 033004 (1998). 
[25] KAMLAND Project, P. Alivisatos et al., preprint Stanford-HEP-98-03, Tohoku-RCNS98-03.

[26] C. Giunti, C. W. Kim, U. W. Lee and V.A. Naumov, hep-ph/990261. 


\section{Figure captions}

Fig. 1. Zenith angle distribution of the excess of the e-like events in the sub-GeV range for

$\sin ^{2} 2 \theta_{23}=0.8, \sin ^{2} 2 \theta_{12}=0.9$ and different values $\Delta m_{21}^{2}$. The points are the 735 days data of the Super-Kamiokande [2].

Fig. 2. Zenith angle distribution of the excess of the e-like events in the sub-GeV range for different values of $\sin ^{2} 2 \theta_{12}$ and for two values of $\Delta m_{21}^{2}=410^{-5} \mathrm{eV}^{2}$ and $210^{-4} \mathrm{eV}^{2}$; $\sin ^{2} 2 \theta_{23}=0.8$. The points are the 735 days data of the Super-Kamiokande [2].

Fig. 3. The same as in Fig.1 for the low energy part of the sub-GeV range $(p<0.4 \mathrm{GeV})$.

Fig. 4. The same as in Fig.1 for the high energy part of the sub-GeV range $(p>0.4 \mathrm{GeV})$.

Fig. 5. The same as in Fig.1 for the multi-GeV range. 


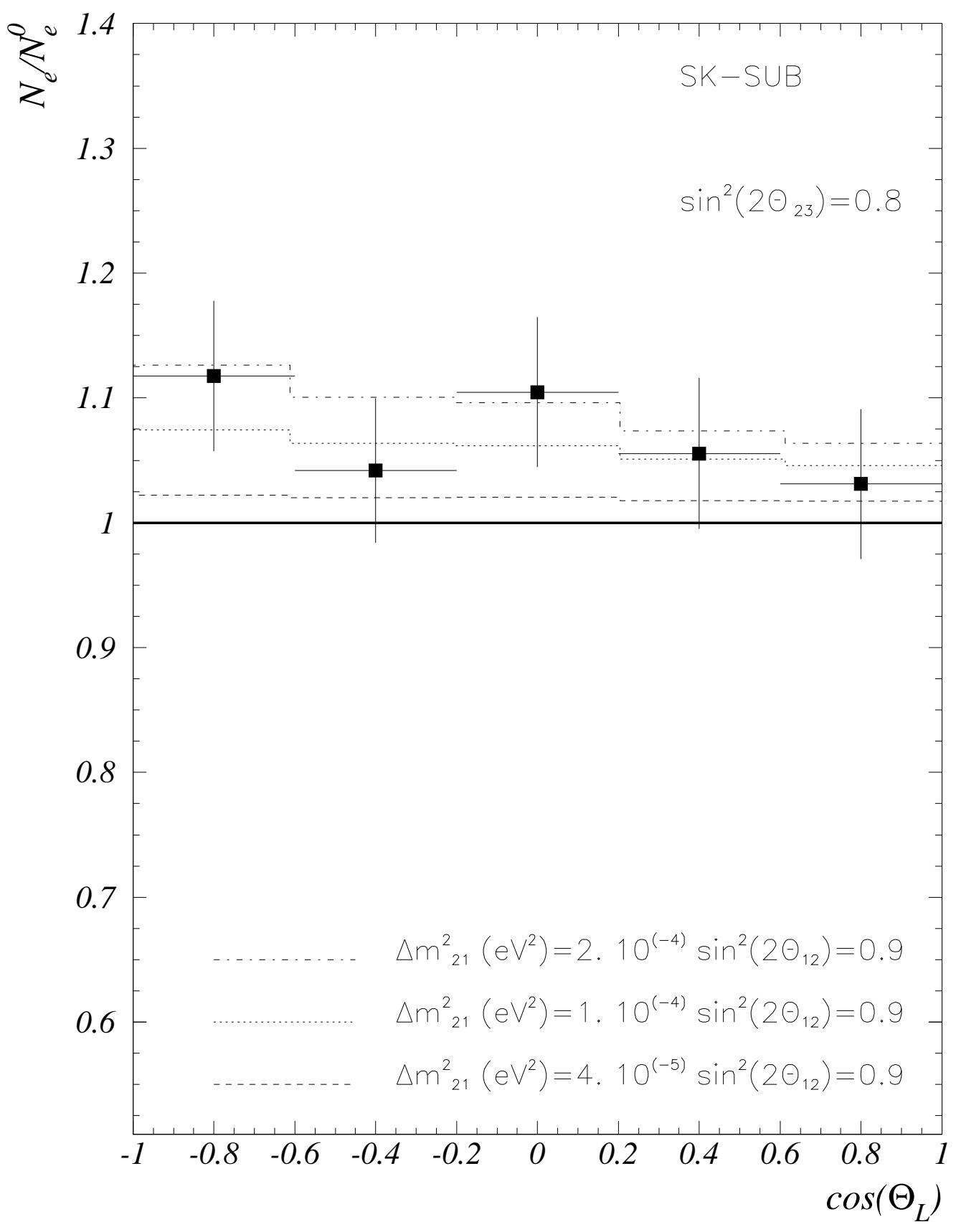

Figure 1: 


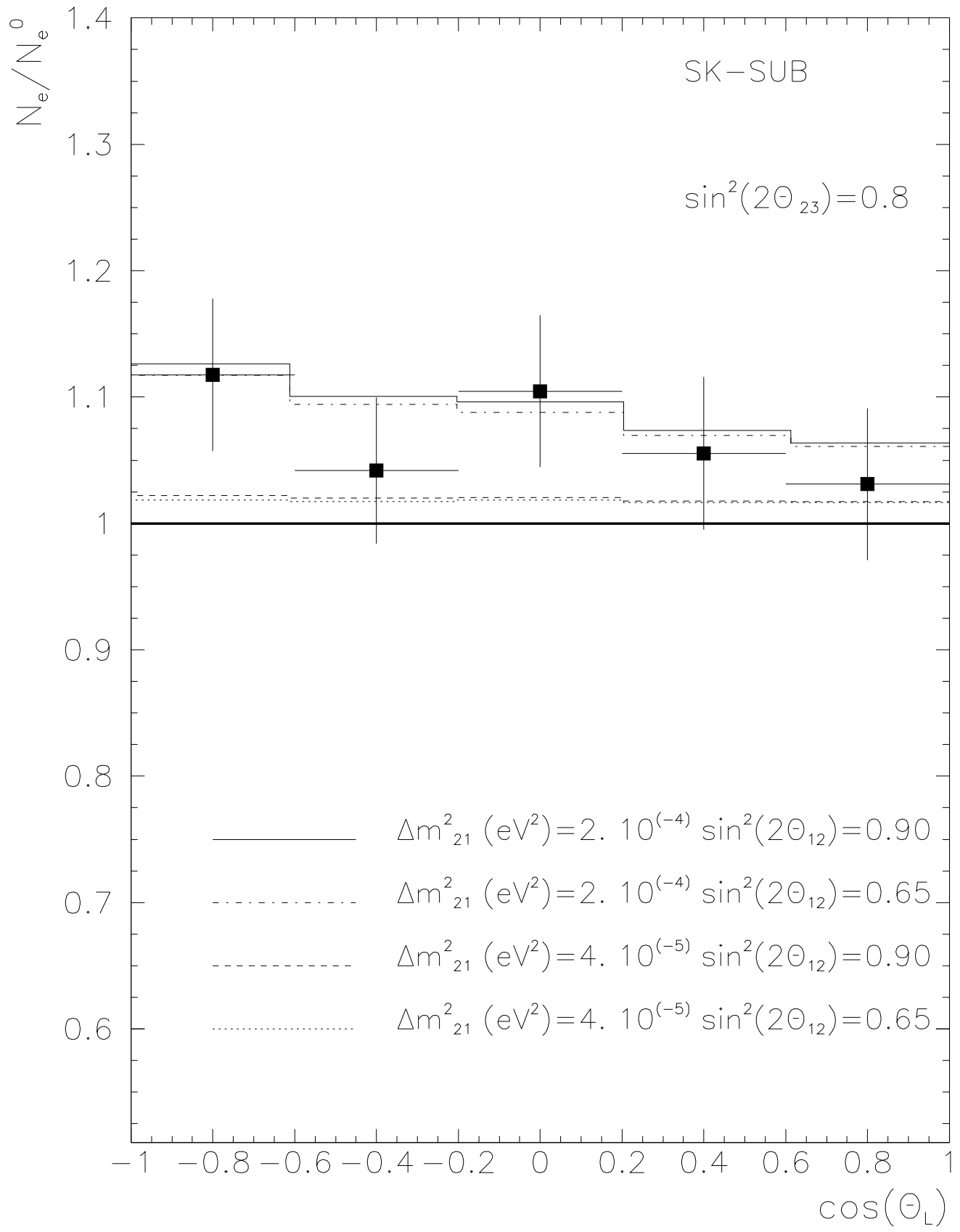

Figure 2: 


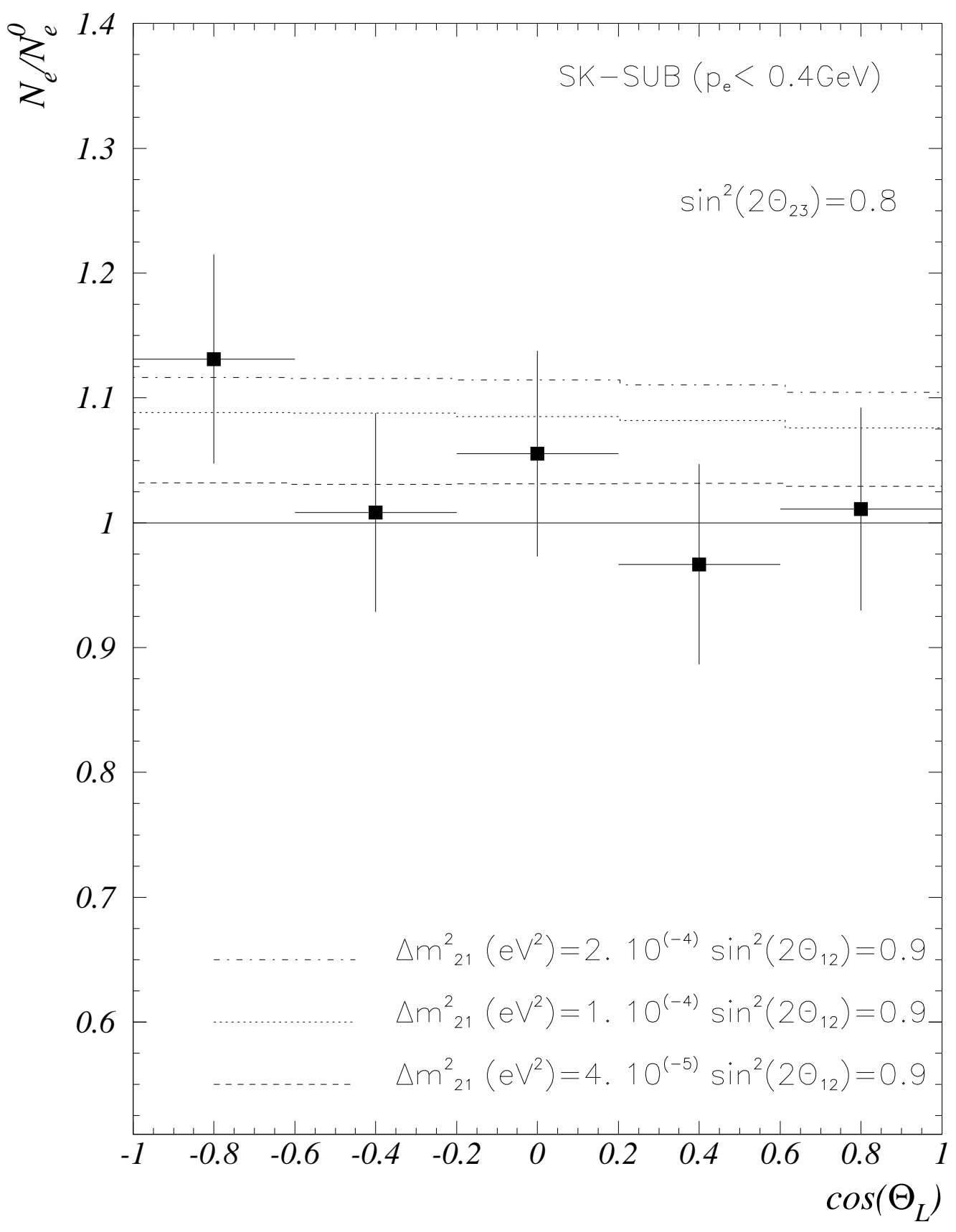

Figure 3: 


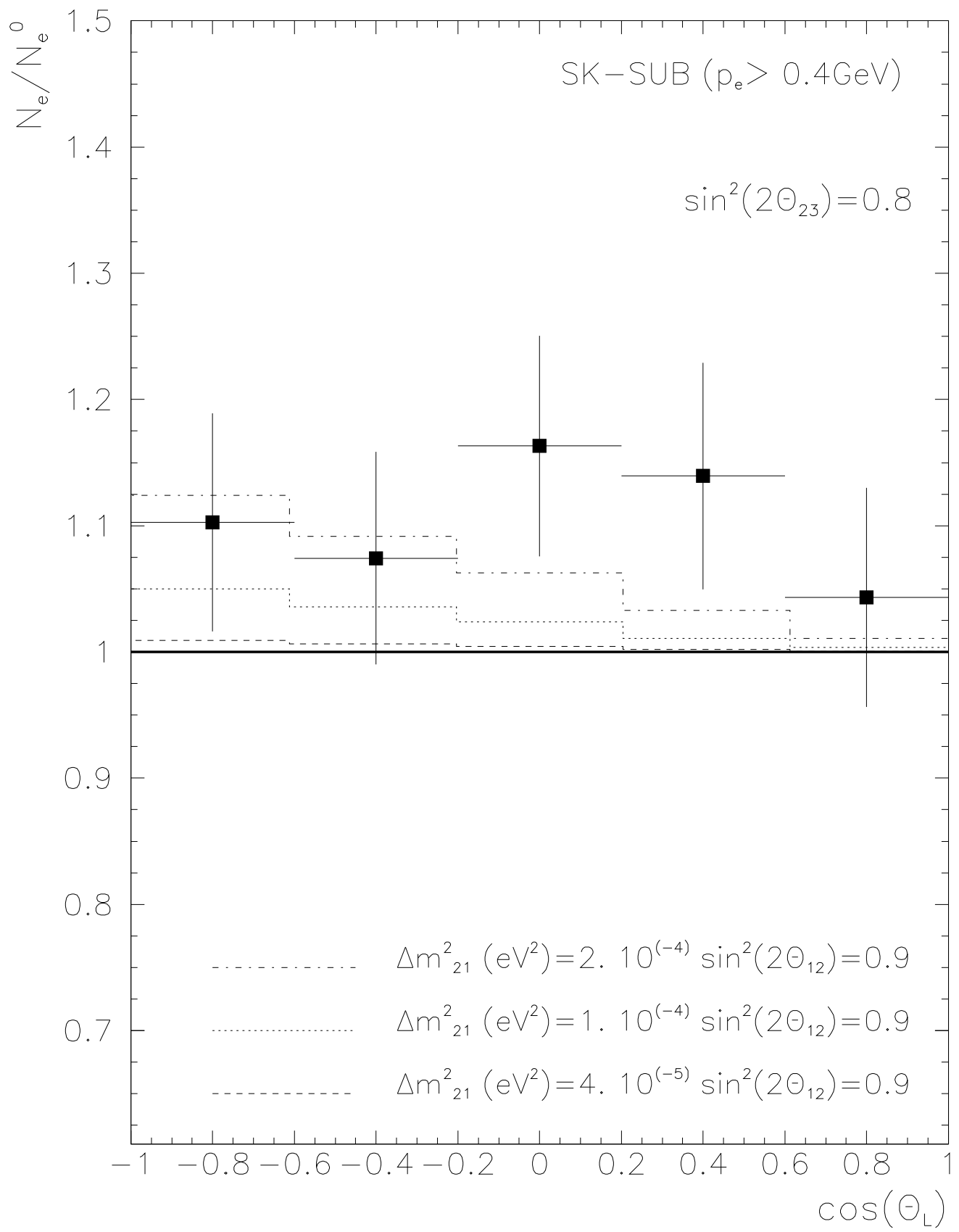

Figure 4: 


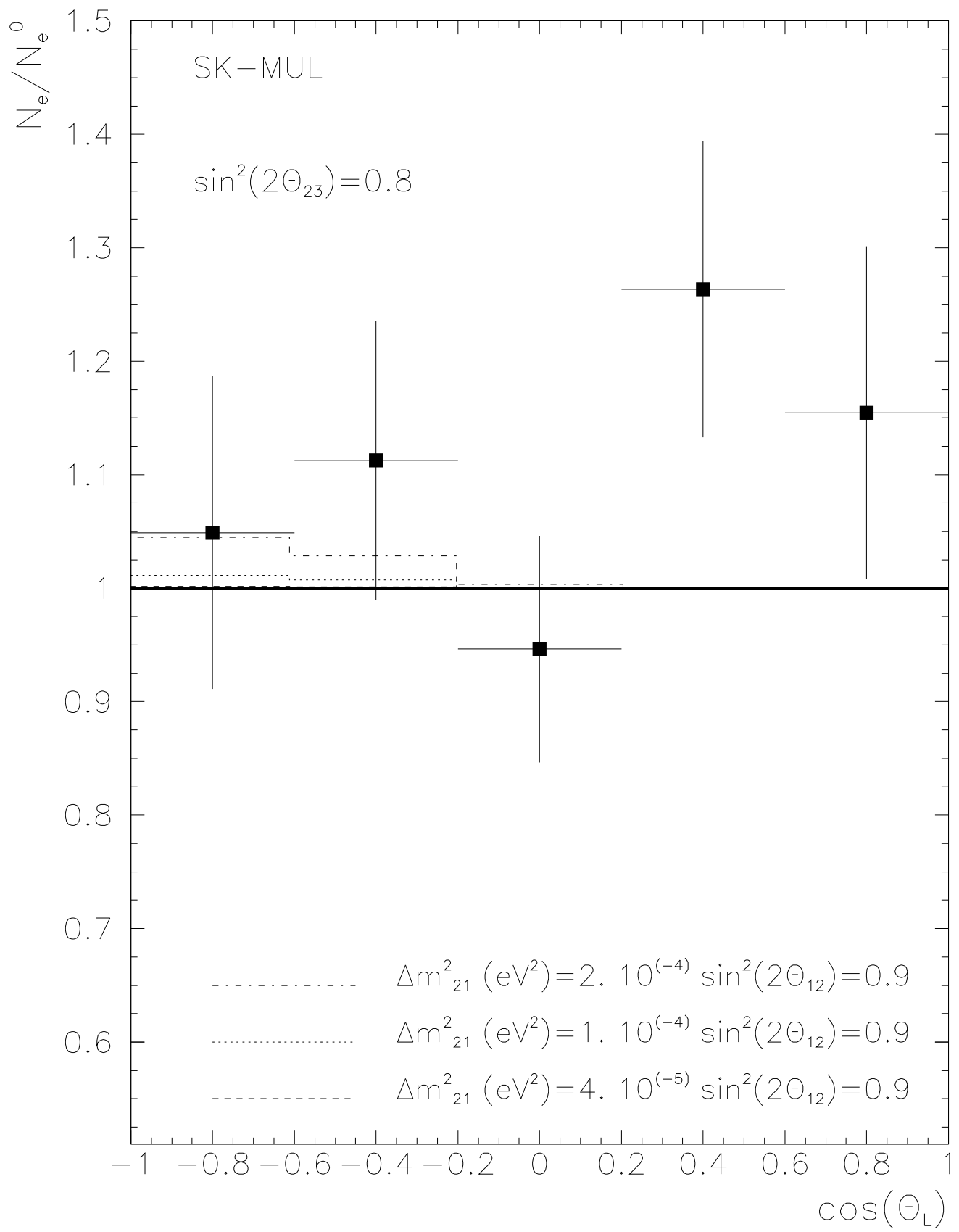

Figure 5: 